\title{
FURTHER CONSIDERATIONS OF THE ELASTIC INCLUSION PROBLEM
}

\author{
by R. J. KNOPS \\ (Received 26th October 1963)
}

\begin{abstract}
Summary
An equation is derived for the strains of an arbitrary elastic field in an infinite matrix perturbed by several inclusions. The equation is solved exactly when the shear moduli of the inclusions and matrix are identical, and also when only a single ellipsoidal inclusion perturbs a field uniform at infinity. Mean-values of the strains are then calculated for non-uniform fields perturbed either by an ellipsoid or by a system of weakly-interacting spheres.
\end{abstract}

\section{Introduction}

Classical elasticity theory is here applied to the determination of the stress and displacement in an infinite isotropic homogeneous matrix containing several isotropic homogeneous inclusions of arbitrary shape and different elastic properties. The inclusions are regarded as being bonded to the matrix, which means that the traction and displacement are continuous across each interface separating an inclusion from the matrix.

To solve the problem we imagine the matrix to be continuously extended throughout the entire space and then determine what force distributions are required in this fictitious medium to produce a displacement field identical to that occurring in the original body. These forces involve the unknown strains of the inclusion problem, but have the advantage of acting in an infinite homogeneous continuous medium. A straightforward integration therefore furnishes a formula for the displacement which can then be used to derive a system of integro-differential equations satisfied by the strains.

The approach is fundamentally similar to one described by Eshelby (2, $3,4)$, but is more direct mathematically and provides a more concise treatment of the general problem.

The basic equations for the strains can be solved exactly in two cases. The first is when the shear moduli of the matrix and inclusions are identical, and then the problem is reduced to one in potential theory. The second case is when the matrix contains only a single inclusion in the shape of an ellipsoid and the stress at infinity is uniform. This is the problem originally considered by Eshelby. However, apart from these two situations, the equations appear difficult to solve exactly, and for this reason we do not attempt to do so here. 
Instead, we employ the basic equations to calculate the mean-value of the strains for non-uniform fields perturbed by an ellipsoidal inclusion, and also by a system of weakly-interacting spherical inclusions of any size and number.

Some other recent contributions in this field of study have been concerned with using variational techniques to derive bounds for the aggregate moduli of multiphased materials possessing arbitrary phase geometry. In this connexion reference should be made to Hashin and Shrikman (5) and Hill $(7,8)$, where in particular bounds are presented for the bulk and shear moduli. Their work does not in the main overlap with the present article which is devoted more to a new derivation of the general exact solution.

We start by recording the field equations satisfied when the matrix is continuous and when it contains the inclusions, and then in Section 3 derive the basic equations for the strains. This section also establishes the discontinuities in the strains across an interface. Section $\mathbf{4}$ describes the two problems which can be solved exactly, and Section 5 discusses mean-values.

\section{Basic formulæ}

Consider first the situation when the matrix is extended continuously through the whole space and is subjected to the boundary conditions of the original inclusion problem. These consist of a body-force, $f$, distributed over finite portions of space (including regions which may subsequently be occupied by an inclusion), together with conditions at infinity, where either the stress or the displacement, or combinations of them, are prescribed. The Cartesian components of the stress, strain and displacement thus induced are denoted respectively by $\sigma_{i j}^{0}$, $e_{i j}^{0}$ and $u_{i}^{0}$, and satisfy

$$
\begin{aligned}
\sigma_{i j, j}^{0}+f_{i} & =0, \quad i, j=1,2,3, \\
e_{i j}^{0} & =\frac{1}{2}\left(u_{i, j}+u_{j, i}\right), \ldots \ldots \\
\sigma_{i j}^{0} & =L^{0}\left(e_{i j}^{0}\right), \quad \ldots \ldots \ldots \ldots
\end{aligned}
$$

where here and in the following the convention is adopted of summing over repeated suffixes, and a comma indicates partial differentiation.

Equation (2.3) is an abbreviation for the usual linear stress-strain law

$$
\sigma_{i j}^{0}=\lambda^{0} e_{k k}^{0} \delta_{i j}+2 \mu^{0} e_{i j}^{0}
$$

in which $\delta_{i j}$ is the Kronecker delta, and $\lambda^{0}, \mu^{0}$ are the Lamé constants of the matrix. These are related to Poisson's ratio, $v^{0}$ by

$$
\lambda^{0}=2 \mu^{0} v^{0} /\left(1-2 v^{0}\right) .
$$

As we shall later determine the strains when the inclusions are present in terms of the strains, $e_{i j}^{0}$, we always assume that equations (2.1), (2.2), (2.3) can be solved subject to the given boundary conditions.

Next consider the matrix when it contains the inclusions. The stress, strain and displacement have now respectively the Cartesian components 
$\sigma_{i j}, e_{i j}, u_{i}$, which, except on an interface, everywhere satisfy

$$
\begin{aligned}
\sigma_{i j, j}+f_{i} & =0, \quad \ldots \ldots \ldots \ldots . . . \\
e_{i j} & =\frac{1}{2}\left(u_{i, j}+u_{j, i}\right) .
\end{aligned}
$$

In the matrix, the stress-strain law is

while in an inclusion it is

$$
\begin{gathered}
\sigma_{i j}=L^{0}\left(e_{i j}\right), \\
\sigma_{i j}=L\left(e_{i j}\right),
\end{gathered}
$$

or equivalently

$$
\sigma_{i j}=\lambda e_{k k} \delta_{i j}+2 \mu e_{i j}
$$

where $\lambda, \mu$ are the Lamé constants of a typical inclusion. The corresponding Poisson's ratio is $v$.

At infinity, the stress and displacement must satisfy the prescribed values. Across an interface, $\Sigma$ there is continuity of traction and displacement, so that if $n=\left(l_{i}\right)$ is the unit outward normal, we have on $\Sigma$,

$$
\left[l_{j} \sigma_{i j}\right]=0, \quad\left[u_{i}\right]=0,
$$

where the square bracket denotes the jump in the enclosed quantity along $\boldsymbol{n}$, e.g.

$$
\left[u_{i}\right]=u_{i} \text { (matrix) }-u_{i} \text { (inclusion). }
$$

We observe that (2.8) do not imply the continuity of all the stress and strain components across $\Sigma$, and indeed in the next section we establish precisely what these discontinuities are.

\section{Equations for the displacement and strain}

Because the operator, $L$, is linear the stress-strain law (2.7) may be rewritten as

$$
\sigma_{i j}=L^{0}\left(e_{i j}\right)+\left(L-L^{0}\right)\left(e_{i j}\right)
$$

We now regard $L^{0}\left(e_{i j}\right)$ as the stress produced by the strain, $e_{i j}$, in an infinite medium whose Lamé constants are everywhere $\lambda^{0}, \mu^{0}$, i.e. the original matrix is extended continuously to fill the whole space. It follows from (2.4), (2.7), and (2.8) that the stress $L^{0}\left(e_{i j}\right)$ is maintained in equilibrium by a layer of surface-force

$$
-\left(L-L^{0}\right)\left(e_{i j}\right) l_{j}
$$

over the interface of each inclusion, together with a body-force,

$$
\left(L-L^{0}\right)\left(e_{i j}\right),{ }_{j},
$$

distributed over the region of each inclusion. In addition to these forces there are also the original boundary conditions to be satisfied, except that where an inclusion is located at infinity the stress is altered by the amount

$$
-\left(L-L^{0}\right)\left(e_{i j}\right)
$$

Thus the strains and displacements arising when the inclusions are present can be reproduced in the matrix when it is continuously extended throughout 
all space, if, besides the original boundary conditions, the forces (3.2) and (3.3) are introduced. Several methods of solution are available. The one we select is the so-called Betti method of integration and requires the following expression for the displacement in the $i$ th direction at the point $P\left(x_{1}, x_{2}, x_{3}\right)$ due to a unit point-force in the $j$ th direction at the point $Q\left(y_{1}, y_{2}, y_{3}\right)$ :

$$
K_{i j}=\frac{1}{4 \pi \mu^{0}} \frac{\delta_{i j}}{R}-\frac{1}{16 \pi \mu^{0}\left(1-v^{0}\right)} R,{ }_{i j},
$$

where

$$
R^{2}=\left(x_{i}-y_{i}\right)\left(x_{i}-y_{i}\right)
$$

and differentiation is with respect to $x_{1}, x_{2}, x_{3}$.

If the matrix is not infinite, then $K_{i j}$ has to be augmented by a regular part $G_{i j}$ which satisfies certain conditions on the boundary of the matrix. For nstance, if the traction is specified there, then $K_{i j}+G_{i j}$ must produce vanishing surface tractions. Because the form of $G_{i j}$ is known for so very few bodies, we do not consider finite problems.

By means of (3.5) we may determine the displacement due to the forcedistributions (3.2) and (3.3) and the boundary conditions of the problem. A simple integration with respect to $Q$ yields

$$
u_{i}(P)=-\int\left(L-L^{0}\right)\left(e_{t j}\right) l_{j} K_{i t} d S+\int\left(L-L^{0}\right)\left(e_{t j}\right),{ }_{j} K_{i t} d v+u_{i}^{0}(P),
$$

where the integrals are over the interfaces and volumes of all the inclusions. On applying the divergence theorem, (3.6) becomes

$$
u_{i}(P)=-\int\left(L-L^{0}\right)\left(e_{i j}\right) K_{i t, j} d v+u_{i}^{0}(P)
$$

or, since

$$
\frac{\partial R}{\partial x_{i}}=-\frac{\partial R}{\partial y_{i}}, \frac{\partial}{\partial x_{i}}\left(\frac{1}{R}\right)=-\frac{\partial}{\partial y_{i}}\left(\frac{1}{R}\right),
$$

we may re-write this equation in the form

$$
u_{i}(P)=\frac{\partial}{\partial x_{j}} \int\left(L-L^{0}\right)\left(e_{t j}(Q)\right) K_{i t}(P, Q) d v(Q)+u_{i}^{0}(P),
$$

where for clarity we exhibit the dependence of the functions on their arguments. Substitution of (3.7) into the strain-displacement relation and use of (3.5) then leads to

$$
\begin{aligned}
e_{i j}(P)=\frac{1}{8 \pi \mu^{0}} & {\left[\frac{\partial^{2}}{\partial x_{t} \partial x_{j}} \int \frac{\left(L-L^{0}\right)\left(e_{i t}\right)}{R} d v+\frac{\partial^{2}}{\partial x_{t} \partial x_{i}} \int \frac{\left(L-L^{0}\right)\left(e_{j t}\right)}{R} d v\right] } \\
& -\frac{1}{16 \pi \mu^{0}\left(1-v^{0}\right)} \frac{\partial^{4}}{\partial x_{i} \partial x_{j} \partial x_{k} \partial x_{t}} \int\left(L-L^{0}\right)\left(e_{k t}\right) R d v+e_{i j}^{0}(P),
\end{aligned}
$$

which represents the main result of the paper. A contraction with respect to 
$i$ and $j$ immediately gives an expression for the dilatation:

$$
e_{k k}(P)=\frac{1}{4 \pi\left(\lambda^{0}+2 \mu^{0}\right)} \frac{\partial^{2}}{\partial x_{i} \partial x_{j}} \int \frac{\left(L-L^{0}\right)\left(e_{i j}\right)}{R} d v+e_{k k}^{0}(P)
$$

Before investigating solutions of equation (3.8) we examine the discontinuity in the strain components across an interface.

These may be derived in two ways. The first uses equation (3.8) and the discontinuity relations for the harmonic and biharmonic potentials. These are given, for instance, by Eshelby (4) and show that:

$$
\left[e_{i j}\right]=\left(\frac{\lambda-\lambda^{0}}{\lambda^{0}+2 \mu^{0}}\right) l_{i} l_{j} e_{k k}^{+}+\left(\frac{\mu-\mu^{0}}{\mu^{0}}\right)\left(e_{i t}^{+} l_{t} l_{j}+e_{j t}^{+} l_{t} l_{i}\right)-\frac{\mu-\mu^{0}}{\mu^{0}\left(1-v^{0}\right)} l_{i} l_{j} l_{k} l_{t} e_{k t}^{+} \text {, }
$$

where the square bracket is defined in the sense of (3.8) and $e_{i j}^{+}$represents the strain on the inclusion side of the interface. The second method is independent of (3.8) and uses the property (see Hill (6)) that the partial derivatives of the continuous displacement can possess only the discontinuities

$$
\left[u_{i, j}\right]=\eta_{i} l_{j},
$$

where $\eta_{i}$ is some function to be determined. The first of (3.8) can be rewritten as

$$
l_{j}\left[L\left(e_{i j}\right)\right]=0
$$

and so by means of a relation of the type

$$
[f g]=f^{+}[g]+g^{+}[f]+[f][g]
$$

we have

$$
\eta_{i}=\left(\frac{\lambda-\lambda^{0}}{\lambda^{0}+2 \mu^{0}}\right) l_{i} e_{k k}^{+}+2\left(\frac{\mu-\mu^{0}}{\mu^{0}}\right) l_{j} e_{i j}^{+}-\frac{2\left(\mu-\mu^{0}\right)}{\mu^{0}\left(1-\nu^{0}\right)} l_{i} l_{k} l_{t} e_{k t}^{+}
$$

which is equivalent to (3.10).

We next consider how equation (3.8) can be solved.

\section{Some solutions}

An exact solution is always possible whenever the shear moduli of the inclusions and matrix are identical, for then

$$
\left(L-L^{0}\right)\left(e_{i j}\right)=\left(\lambda-\lambda^{0}\right) e_{k k} \delta_{i j},
$$

and so from equation (3.9), we have in an inclusion,

$$
e_{k k}=\left(\frac{\lambda^{0}+2 \mu^{0}}{\lambda+2 \mu^{0}}\right) e_{k k}^{0}
$$

and in the matrix,

$$
e_{k k}=e_{k k}^{0} \text {. }
$$

The dilatation is therefore directly proportional to the unperturbed value even though this is non-uniform. The strain is obtained by substituting (4.1) 
into (3.8) and is

$$
e_{i j}=\frac{\left(\lambda-\lambda^{0}\right)}{4 \pi\left(\lambda+2 \mu^{0}\right)} \frac{\partial^{2}}{\partial x_{i} \partial x_{j}} \int \frac{e_{k k}^{0}}{R} d v+e_{i j}^{0}
$$

with the displacement being given by

$$
\boldsymbol{u}=\frac{\left(\lambda-\lambda^{0}\right)}{4 \pi\left(\lambda+2 \mu^{0}\right)} \operatorname{grad} \int \frac{e_{k k}^{0}}{R} d v+u^{0} .
$$

The problem thus reduces to the determination of a known gravitational distribution, $e_{k k}^{0}$, over the region of the inclusions. Moreover, the effect of the inclusions is linear since by (4.1) and (4.2) they do not interact with each other, and so to find, for instance, the displacement due to several inclusions we simply add together displacements due to each inclusion acting alone. It is also worthwhile noting that the discontinuities (3.10) simplify considerably and with the help of (4.1) can be made to depend entirely upon the known unperturbed dilatation. These conclusions have been previously derived by the present author (10) using a different approach.

Hill $(7,8)$ has also solved this problem for a body loaded so as to produce a constant dilatation in the matrix and inclusion. The method utilises a semiinverse procedure in potential theory.

Probably the most obvious manner of solving the basic equation (3.8) is to use an iterative procedure in which the $n$th approximation, $e_{i j}^{(n)}$, to the strain, $e_{i j}$, is given by the recurrence relation

$$
e_{i j}^{(n)}=H\left\{e_{i j}^{(n-1)}\right\}+e_{i j}^{0}
$$

where $H$ stands for the operator on the right-hand of (3.8). In general it would appear rather lengthy to evaluate the strains in this way, but there is one notable exception in which the iterative procedure leads to an algebraic system of equations. This is for a single ellipsoidal inclusion perturbing a stress uniform at infinity, and with no body-force present. Then, by virtue of the property of harmonic and biharmonic potentials for an ellipsoid, all the approximations to the strains within the inclusion are constant, and so we must have that the inclusion strains satisfy

$$
\begin{aligned}
e_{i j}=\frac{1}{8 \pi \mu^{0}}\left[\left(L-L^{0}\right)\left(e_{t i}\right) \phi_{, t j}+\right. & \left.\left(L-L^{0}\right)\left(e_{t i}\right) \phi_{, t i}\right] \\
& -\frac{1}{16 \pi \mu^{0}\left(1-v^{0}\right)}\left(L-L^{0}\right)\left(e_{k t}\right) \psi_{, i j k t}+e_{i j}^{0} .
\end{aligned}
$$

Here, $\phi$ and $\psi$ are respectively the harmonic and biharmonic potentials of a gravitational distribution of unit density over the ellipsoidal inclusion.

Eshelby solved this problem $(2,3,4)$ in a somewhat less direct manner than the one adopted here. In (4), he determined the various partial derivatives of $\phi, \psi$ that enter into (4.3), and with the help of these the complete solution can be derived. To avoid duplication, we do not record the solution. However, 
it is interesting to observe that the shear strain within the inclusion is directly proportional to the corresponding unperturbed shear strain, while the normal strain-components are influenced by all components of the unperturbed normal strains in rather a complicated way.

We now leave aside the question of the exact solution of (3.8) and next consider mean-values of the strains for ellipsoidal and spherical inclusions in a non-uniform field.

\section{Mean-values of the strains}

We start again with the simple problem of the single ellipsoidal inclusion and integrate both sides of equation (3.8) over the inclusion to obtain

$$
\begin{aligned}
\bar{e}_{i j}=\frac{1}{8 \pi \mu^{0}}\left[\left(L-L^{0}\right)\left(\bar{e}_{t j}\right) \phi_{, t i}+\right. & \left.\left(L-L^{0}\right) \bar{e}_{t i} \phi_{, t j}\right] \\
& -\frac{1}{16 \pi \mu^{0}\left(1-v^{0}\right)}\left(L-L^{0}\right)\left(\bar{e}_{k t}\right) \psi_{, i j k t}+\bar{e}_{i j}^{0},
\end{aligned}
$$

where $\phi$ and $\psi$ are the potentials of (4.3), and the bar indicates an integral over the inclusion. Equations (5.1) are identical in structure to those of (4.3) and therefore have the same solution. We observe, however, that now no restriction is placed upon the unperturbed strain.

In the special case of a spherical inclusion, the perturbed and unperturbed volume expansions are related by

$$
\left(3 \lambda+2 \mu+4 \mu^{0}\right) \bar{e}_{k k}=3\left(\lambda^{0}+2 \mu^{0}\right) \bar{e}_{k k}^{0} \text {. }
$$

When no body-force acts within the inclusion, the dilatations $e_{k k}, e_{k k}^{0}$ are both harmonic there, and hence by the classical mean-value theorem for a sphere, equation (5.2) may be replaced by

$$
\left(3 \lambda+2 \mu+4 \mu^{0}\right) e_{k k}(C)=3\left(\lambda^{0}+2 \mu^{0}\right) e_{k k}^{0}(C),
$$

where $C$ is the centre of the sphere. The remaining mean-values of the strains are given by

$$
\bar{e}_{i j}=\alpha \bar{e}_{k k}^{0} \delta_{i j}+\beta \bar{e}_{i j}^{0}
$$

where

$$
\alpha=\frac{3\left[5\left(\lambda-\lambda^{0}\right)\left(2 v^{0}-1\right)+2\left(\mu-\mu^{0}\right)\right]\left(\lambda^{0}+2 \mu^{0}\right)}{\left[\mu^{0}\left(7-5 v^{0}\right)-2 \mu\left(5 v^{0}-4\right)\right]\left(3 \lambda+2 \mu+4 \mu^{0}\right)}, \quad \beta=\frac{15 \mu^{0}\left(1-v^{0}\right)}{\mu^{0}\left(7-5 v^{0}\right)-2 \mu\left(5 v^{0}-4\right)} .
$$

Again, let no body-force act in the inclusion. Then $e_{i j}, e_{i j}^{0}$, are biharmonic there, and their mean-values can be replaced by their values at the centre as follows. The solid mean-value theorem for biharmonic functions (1) gives

$$
\frac{3}{4 \pi a^{3}} \bar{e}_{i j}=e_{i j}(C)+\frac{a^{2}}{10} e_{i j, k k}(C)
$$


where $a$ is the radius of the sphere. From the Navier equations,

we easily deduce that

$$
\mu u_{i, k k}+(\lambda+\mu) u_{k, k i}=0 \text {, }
$$

$$
e_{i j, k k}=-\frac{(\lambda+\mu)}{\mu} e_{k k, i j}
$$

and with another application of the harmonic mean-value theorem we get

$$
e_{i j, k k}(C)=-\frac{3(\lambda+\mu)}{4 \pi \mu a^{3}} \int e_{k k, i j} d v \text {. }
$$

But, by (3.9), and remembering that $\phi_{, i j k t}=0$, we have

$$
\int e_{k k, i j} d v=\int e_{k k, i j}^{0} d v
$$

Hence, by a further application of the harmonic mean-value theorem we arrive at

$$
\bar{e}_{i j}=\frac{4 \pi a^{3}}{3} e_{i j}(C)-\frac{2 \pi a^{5}}{15} \frac{\lambda+\mu}{\mu} e_{k k, i j}^{0}(C),
$$

and finally an elimination of $\bar{e}_{i j}$ between this equation and (5.4) gives $e_{i j}(C)$ in terms of the known strains $e_{i j}^{0}$ :

$$
e_{i j}(C)=\alpha e_{k k}^{0}(C) \delta_{i j}+\beta e_{i j}^{0}(C)+\frac{a^{2}}{10}\left[\frac{\lambda+\mu}{\mu}-\frac{\lambda^{0}+\mu^{0}}{\mu}\right] e_{k k, i j}^{0}(C) .
$$

Once having found the mean-values of the strains, we can immediately determine the displacement at large distances from the inclusion by means of MacCullagh's formula applied to equation (3.7). Thus, for $P$ at a large distance we have,

$$
u_{i}(P)=\frac{\left(L-L^{0}\right)\left(\bar{e}_{i j}\right)}{4 \pi \mu^{0}} \frac{\partial}{\partial x_{j}}\left(\frac{1}{R}\right)-\frac{1}{16 \pi \mu^{0}\left(1-v^{0}\right)}\left(L-L^{0}\right)\left(\bar{e}_{i j}\right) R,,_{i j t}+u_{i}^{0}(P), \ldots
$$

where $R$ is now the distance between $P$ and the centre of the ellipsoid. If the unperturbed field $\boldsymbol{u}^{0}$ corresponds to a pure dilatation, then the above result shows that at large distances the inclusion may be replaced by a centre of dilatation acting at its centre. If $\boldsymbol{u}^{0}$ corresponds to a pure shear, then the displacement (5.7) is appropriate to a double force placed at the centre of the inclusion.

As a second application consider a system of spherical inclusions which weakly-interact with each other, i.e. the interaction is a first-order effect. To examine more closely what this approximation implies we treat first of all the problem when only two inclusions are present. Let their centres be a distance, $\Gamma$, apart and let their line of centres be along the 3-axis. Further, let $\left(x_{i}\right)$ and $\left(y_{i}\right)$ be respectively the coordinates relative to the centre of two points situated in sphere 1 and sphere 2. The distance, $R$, between these points is then,

$$
R^{2}=R_{1}^{2}-2 x_{i} y_{i}+2 y_{3} \Gamma
$$


where $R_{1}$ is given by

$$
R_{1}^{2}=x_{i} x_{i}+\Gamma^{2}-2 \Gamma x_{3}
$$

and so is the distance between $\left(x_{i}\right)$ and the centre of the sphere 2 .

To solve the problem, we apply (3.8) with $P$ taken at the point $\left(x_{i}\right)$ of the sphere 1 , and by the multinomial theorem expand in terms of $\Gamma$ the values of $R^{-1}$ and $R$ which occur in the integrals over the sphere 2. Thus, for the harmonic potential integral, we use (5.8) to obtain,

$$
\begin{aligned}
\frac{\partial^{2}}{\partial x_{t} \partial x_{j}} \int_{2} \frac{\left(L-L^{0}\right)\left(e_{t i}\right)}{R} d v & =\frac{\partial^{2}}{\partial x_{t} \partial x_{j}} \int_{2} \frac{1}{R_{1}}\left[1+\frac{x_{i} y_{i}-y_{3} \Gamma}{R_{1}^{2}}+\ldots\right]\left(L-L^{0}\right)\left(e_{t i}\right) d v \\
& =\frac{\partial^{2}}{\partial x_{t} \partial x_{j}}\left\{\frac{1}{R_{1}}\right\} \int_{2}\left(L-L^{0}\right)\left(e_{t i}\right) d v+O\left(R_{1}^{-4}\right), \ldots \ldots \ldots .(5
\end{aligned}
$$

where it is assumed that the strain, $e_{i j}$, is bounded. Upon carrying out the differentiation in (5.10), and then expanding $R^{-3}, R^{-5}$ in terms of $\Gamma$ by means of (5.9), we see that the right-side of (5.10) reduces to

$$
-\Gamma^{-3} \int_{2}\left(L-L^{0}\right)\left(e_{i j}\right) d v+O\left(\Gamma^{-4}\right) \text {. }
$$

Similarly, it may be shown that

$$
\begin{aligned}
& \frac{\partial^{4}}{\partial x_{i} \partial x_{j} \partial x_{k} \partial x_{t}} \int_{2} R\left(L-L^{0}\right)\left(e_{k t}\right) d v \\
& \quad=-\left(\delta_{k t} \delta_{i j}+\delta_{k i} \delta_{t j}+\delta_{k j} \delta_{t i}\right) \Gamma^{-3} \int_{2}\left(L-L^{0}\right)\left(e_{k t}\right) d v+O\left(\Gamma^{-4}\right) .
\end{aligned}
$$

The approximation consists in neglecting the $O\left(\Gamma^{-4}\right)$ terms in (5.11) and (5.12).

The method can obviously be applied to any number of spheres, but the calculations are rather cumbersome. For simplicity, therefore, we treat only the following problem in which the integrals of the strains over the inclusion are assumed the same. Consider an infinite plane square array of equal spherical inclusions, of radii $a$, and whose base square is of side $p$. Select the origin of coordinates to be at the centre of any sphere, and take the point, $P$, of (3.8) to be in this sphere. Substitution of (5.11) and (5.12) for the appropriate integrals over the remaining spheres that arise in (3.8), and then integration over the base sphere leads to a system of equations whose solution is

where

$$
M \bar{e}_{i j}=\tilde{e}_{i j}^{0}+\{N /(M-3 N)\} \bar{e}_{k k}^{0} \delta_{i j},
$$

$$
\begin{aligned}
& M=1+2\left(\mu-\mu^{0}\right)\left(4-5 v^{0}\right) / 15 \mu^{0}\left(1-v^{0}\right) \\
& -\left[\left(\mu-\mu^{0}\right)\left(1-2 v^{0}\right) a^{3} /\left\{3 \mu^{0}\left(1-v^{0}\right) p^{3}\right\}\right] \sum_{l, m}\left(l^{2}+m^{2}\right)^{-\frac{3}{2}}, \\
& \begin{array}{r}
N=\left[2\left(5 v^{0}-4\right)\left(\lambda-\lambda^{0}\right)+3\left(\kappa-\kappa^{0}\right)\right] / 30 \mu^{0}\left(1-v^{0}\right) \\
+\left\{\left[3\left(\kappa-\kappa^{0}\right)-\left(1-2 v^{0}\right)\left(\lambda-\lambda^{0}\right)\right] a^{3} /\left\{6 \mu^{0}\left(1-v^{0}\right) p^{3}\right\}\right\} \sum_{i, m}\left(l^{2}+m^{2}\right)^{-\frac{3}{2}},
\end{array}
\end{aligned}
$$


and we have introduced the bulk modulus, $\kappa$, defined by

$$
3 \kappa=(3 \lambda+2 \mu),
$$

with a corresponding definition for $\kappa^{0}$.

The summation entering into the expressions for $M, N$, is over all values of $l, m$, in $(-\infty, \infty)$, except $l=m=0$. It has been evaluated by Van der Hoff and Bensen (9) who give the value 9.0336. They have also evaluated (9) the sum corresponding to a hexagonal array, and give the value 11.0342.

It should be observed that we cannot apply this treatment to a threedimensional array because the sums involved are divergent.

\section{Note added in proof}

Since this manuscript was prepared, a monograph by V. D. Kupradze (Progress in Solid Mechanics, Vol. 3, 1963) has appeared. This concerns a basic treatment of the inclusion problem in both the static and dynamic cases for finite and infinite matrices. It deals extensively with the existence and uniqueness of the solution to the equations corresponding to our (3.8), and discusses their numerical solution.

\section{REFERENCES}

(1) R. Courant, Methods of Mathematical Physics (New York, 1962), p. 288.

(2) J. D. EsHelby, The determination of the elastic field of an ellipsoidal inclusion and related problems, Proc. Roy. Soc. A, 241 (1957), 376.

(3) J. D. Eshelby, The elastic field outside an ellipsoidal inclusion, Proc. Roy. Soc. $A, 252$ (1959), 561.

(4) J. D. Eshelby, Elastic inclusions and inhomogeneities, Progress in Solid Mechanics, 2 (1961), 89.

(5) Z. Hashin and S. Shrikman, A variational approach to the theory of the elastic behaviour of multiphase materials, J. Mech. Phys. Solids, 11 (1963), 127.

(6) R. HILL, Discontinuity relations in mechanics of solids, Progress in Solid Mechanics, 2 (1961), 247.

(7) R. HILl, Report on theories of the elastic properties of reinforced solids, BISRA Report P/19/62 (1962).

(8) R. HiLl, New derivations of some elastic extremum principles, J. Mech. Phys. Solids, 11 (1963), 357.

(9) B. M. E. VAN Der Hoff and G. C. Benson, A method for the evaluation of some lattice sums occurring in calculations of physical properties of crystals, Can. J. Phys., 31 (1953), 1087.

(10) R. J. KNops, On the use of Poisson's ratio in studying certain non-homogeneous elastic inclusions, Z.A.M.M., 40 (1960), 542.

DepartMent of MATHEMatics

UNIVERSITY OF NEWCASTLE UPON TYNE 